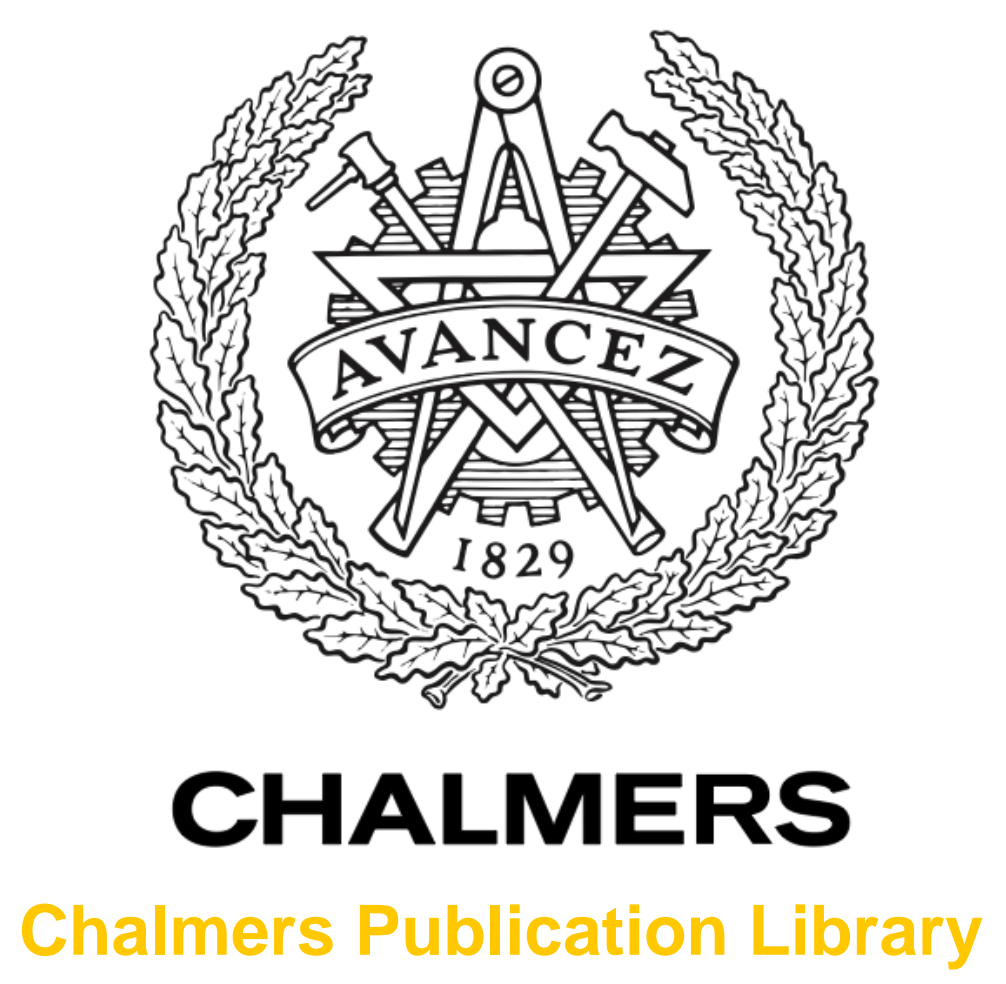

A Study on Engine Health Monitoring in the Frequency Domain

This document has been downloaded from Chalmers Publication Library (CPL). It is the author's version of a work that was accepted for publication in:

Journal of Engineering for Gas Turbines and Power-Transactions of the Asme (ISSN: 07424795)

Citation for the published paper:

Borguet, S. ; Henriksson, M. ; McKelvey, T. (2011) "A Study on Engine Health Monitoring in the Frequency Domain". Journal of Engineering for Gas Turbines and Power-Transactions of the Asme, vol. 133(8),

http://dx.doi.org/10.1115/1.4002832

Downloaded from: http://publications.lib.chalmers.se/publication/140606

Notice: Changes introduced as a result of publishing processes such as copy-editing and formatting may not be reflected in this document. For a definitive version of this work, please refer to the published source. Please note that access to the published version might require a subscription.

Chalmers Publication Library (CPL) offers the possibility of retrieving research publications produced at Chalmers University of Technology. It covers all types of publications: articles, dissertations, licentiate theses, masters theses, conference papers, reports etc. Since 2006 it is the official tool for Chalmers official publication statistics. To ensure that Chalmers research results are disseminated as widely as possible, an Open Access Policy has been adopted.

The CPL service is administrated and maintained by Chalmers Library. 


\section{A STUDY ON ENGINE HEALTH MONITORING IN THE FREQUENCY DOMAIN}

\author{
S. Borguet \\ Turbomachinery Group \\ University of Liège \\ Liège, Belgium \\ Email: s.borguet@ulg.ac.be \\ T. McKelvey \\ Signals and Systems \\ Chalmers University of technology \\ Göteborg, Sweden \\ Email:tomas.mckelvey@chalmers.se
}

\author{
M. Henriksson \\ Performance \& Control systems \\ Volvo Aero Corporation AB \\ Trollhättan, Sweden \\ Email: mattias.mh.henriksson@volvo.com
}

\author{
O. Léonard \\ Turbomachinery Group \\ University of Liège \\ Liège, Belgium \\ Email: o.leonard@ulg.ac.be
}

\section{ABSTRACT}

Most of the techniques developed so far for module performance analysis rely on steady-state measurements from a single operating point to evaluate the level of deterioration of an engine. One of the major difficulties associated with this estimation problem comes from its underdetermined nature. It results from the fact that the number of health parameters exceeds the number of available sensors. Among the panel of remedies to this issue, a few authors have investigated the potential of using data collected during a transient operation of the engine. A major outcome of these studies is an improvement in the assessed health condition.

The present contribution proposes a framework that formalises this observation for a given class of input signals. The analysis is performed in the frequency domain, following the lines of system identification theory. More specifically, the meansquared estimation error is shown to drastically decrease when using transient input signals. The study is conducted with an engine model representative of a commercial turbofan.

Keywords: Gas path analysis, frequency domain, least-squares, system identification.

\section{NOMENCLATURE}

E(.) Mathematical expectation operator

FIM Fisher Information Matrix

$j \quad$ Imaginary symbol $(\sqrt{-1})$

$N \quad$ Spool speed

$P \quad$ Covariance matrix

$P i \quad$ Total pressure at station $i$

$p \quad$ Time differentiation operator

$S E i \quad$ Scaling factor on component efficiency, reference value is 1.0

$S W i$ Scaling factor on component flow capacity, reference value is 1.0

$T i \quad$ Total temperature at station $i$

$W F \quad$ Fuel flow

$x \quad$ State vector

$y \quad$ Measurement signal vector

$u \quad$ Input signal vector

$\alpha \quad$ Amplitude of the sinusoids

$\varepsilon \quad$ Measurement disturbance

$\omega \quad$ Frequency of the sinusoids

$\theta \quad$ Health parameter vector 


\author{
$\theta_{\star} \quad$ Actual value of the health parameters \\ $\hat{\imath} \quad$ Estimate \\ * Conjugate transpose operator \\ $\mathcal{N}(m, C)$ the Gaussian probability density function with \\ mean $m$ and covariance matrix $C$
}

\section{INTRODUCTION}

Predictive maintenance aims at scheduling overhaul actions on the basis of the actual level of deterioration of the engine. The benefits are improved dispatch reliability and safety as well as reduced life cycle costs. Generating reliable information about the health condition of the gas turbine is therefore a requisite and has been the subject of intensive research in the community.

In this paper, Module Performance Analysis is considered. Its purpose is to detect, isolate and quantify the changes in engine module performance, described by so-called health parameters, on the basis of measurements collected along the gas path of the engine [1]. Typically, the health parameters are correcting factors on the efficiency and flow capacity of the modules (fan, LPC, HPC, HPT, LPT) while the measurements are intercomponent temperatures, pressures, shaft speeds and fuel flow. Since the pioneering work by Urban [2], most of the literature on module performance analysis has considered the processing of data observed during steady-state operation of the engine.

In this framework, the estimation of the health parameters can be cast as an optimisation problem which is characterised by a number of difficulties. The underlying process is non-linear, engine measurements are subject to noise and bias and the introduction of model inaccuracy is inevitable. Moreover, the number of sensors is usually smaller than the number of health parameters, making the problem underdetermined.

One obvious way to tackle this non-uniqueness in the solution would be to complement the sensor set installed on the engine, but significant additional costs would be incurred. Hence, this solution on the hardware side is disregarded by engine manufacturers. Two roads of remedy on the software side have then emerged. The first approach is to make use of a priori information on the parameters, e.g. [3,4]. This strategy, also interpreted as regularisation, directs the estimation algorithm to one particular solution based on a prior knowledge on the health condition of the engine. The second option is to extend the analysis to a multiple operating point formulation [5,6]. This second solution takes advantage of the non-linearity of the engine with respect to its operating point to increase the information contained in the data samples. Yet, stabilisation of the engine at a number of operating points is rather unlikely in flight and turns out to be quite a long process on the testbed.

In the late 80's, Merrington [7] showed that alterations in the health condition are also reflected in measurements collected during transient operation of the engine. His work triggered an endeavour to develop diagnosis techniques based on transient data. The first contributions, e.g. [7,8] intended to derive fault signatures and to detect gross modifications in the dynamic characteristics of the engine. Luppold [9] then applied a Kalman filter to assess the changes in module performance from transient data.

These last years, the definition of the transient diagnosis problem has been steadily refined and the effectiveness of various techniques has been investigated. They encompass leastsquares estimation $[10,11]$, artificial neural networks and genetic algorithms $[12,13]$ for batch treatment of the data, or Kalman filters $[14,15]$ in a recursive framework.

A major outcome of these studies is an improvement in the estimated health condition with respect to the results obtained with steady-state data. Most authors relate this improvement to a better information content of transient data than steady-state one, yet no formal argument has ever supported this observation.

The present contribution attempts to propose such an argument for a given class of transient signals. The analysis is performed in the frequency domain, following the lines of system identification theory. More specifically, the mean-squared estimation error is shown to drastically decrease when using transient input signals. The study is illustrated with an engine model representative of a commercial, high by-pass ratio turbofan.

\section{STATEMENT OF THE PROBLEM}

The scope of this section is to present the theoretical foundation of the methodology developed to show the benefit of using transient signals for diagnosis. First, the model relating the observations to the health parameters is described, in the time and frequency domains. The problem of estimating the health parameters in the frequency domain is then formulated from a system identification standpoint. Finally, metrics to assess the quality of the estimates from transient data are defined.

\section{The engine model in time and frequency domains}

In the framework of module performance analysis, the engine model relating the measurements to the health parameters is basically a non-linear aerothermodynamic model based on mass, energy and momentum conservation laws applied to the engine components. Such a model is naturally expressed in the statespace form, namely:

$$
\begin{aligned}
& \dot{x}=f(x, u, \theta) \\
& y=g(x, u, \theta)
\end{aligned}
$$

where $u$ are the known inputs, defining the operating point of the engine (e.g. fuel flow, flight Mach number, altitude), $\theta$ are the aforementioned health parameters and $x$ are the state variables, associated to the transient effects taking place in the gas path of the engine. Generally speaking, these transient effects belong to three categories, namely the heat transfers between the gas 
path and the components of the engine, the shaft inertia and the fluid transport delays. The first equation describes the dynamic behaviour of the engine, while the second one generates the gaspath measurements $y$.

Let $u_{0}, \theta_{0}, x_{0}$ and $y_{0}$ define a stationary operating point (i.e. $\dot{x}_{0}=0$ ) of the engine. The model can be linearised around this point as:

$$
\begin{aligned}
& \dot{x}=A x+B u \\
& y=C x+D u
\end{aligned}
$$

where the system matrices $A, B, C, D$ have the same definition as in [16]. From now on, $u, x, y$ and $\theta$ represent, with some abuse of notation, deviations with respect to the linearisation point. Note that this "nominal model" does not account for possible changes in the health parameters.

In the frequency domain, a (totally) equivalent representation of system (2) is the so-called transfer function, that describes the linear mapping between the Fourier transform of the inputs and the outputs:

$$
G_{u}(j \omega)=C\left(j \omega I_{n_{x}}-A\right)^{-1} B+D
$$

where $I_{n_{x}}$ is the identity matrix the size of which is the number of state variables $n_{x}$.

Inclusion of the health parameters in the linearised model can be done in two ways. On one hand, they can be introduced as additional states. Their effect on the state derivatives and the measurements is captured through two complementary system matrices, leaving the $A, B, C, D$ matrices of system (2) unaltered. This methodology is the traditional one used in the community (see $[9,14]$ ). The other possibility is to integrate the effect of the health parameters directly into the system matrices. This approach is as relevant as the first one. Indeed, deterioration modifies the engine's behaviour, which is here represented by these matrices. This second formulation is more common in system identification applications and is retained here for its convenience with respect to the objective of this paper. In conclusion the parameter-dependent model writes:

$$
\begin{aligned}
& \dot{x}=\Delta_{A}(\theta) x+\Delta_{B}(\theta) u \\
& y=\Delta_{C}(\theta) x+\Delta_{D}(\theta) u
\end{aligned}
$$

where the deviation from the nominal model $A, B, C, D$ is approximated as linear in the health parameter vector $\theta$ and $\Delta_{M}(\theta)$ is a notation for $M+\sum_{i=1}^{n_{\theta}} M_{i} \theta_{i}$. Note that for the parameterdependent model, the stationary values $x_{0}$ and $y_{0}$ are also affected by the health parameters (just as in classic steady-state analysis).
The corresponding transfer function is given by:

$$
G_{u}(j \omega, \theta)=\Delta_{C}(\theta)\left(j \omega I_{n_{x}}-\Delta_{A}(\theta)\right)^{-1} \Delta_{B}(\theta)+\Delta_{D}(\theta)
$$

Introducing the time differentiation operator $p$ (see [17]), the concept of transfer function can be exported to the time domain.

$$
\begin{array}{ccc}
\text { Time domain } & & \text { Frequency domain } \\
G_{u}(p, \theta) & \leftrightarrow & G_{u}(j \omega, \theta)
\end{array}
$$

\section{Parameter estimation in the frequency domain}

The transfer function (5) provides a deterministic description of the turbine engine. However, the observed data are contaminated with measurement noise whose effect should be incorporated into the model:

$$
y(t)=G_{u}(p, \theta) u(t)+e(t)
$$

where $e(t)$ is a vector of zero-mean, independent and identicallydistributed random variables. As explained in [18], the model can be scaled so that $e(t) \in \mathcal{N}\left(0, I_{n_{y}}\right)$.

The present study focuses on a particular type of input signals $u(t)$, the so-called multi-sine signals:

$$
u(t)=u_{0} \cdot \sum_{i=1}^{n_{\omega}} \alpha_{i} \cos \left(\omega_{i} t+\phi_{i}\right)
$$

Multisines have been used to a large extent for system identification, see for instance $[19,20]$ for some applications to turbine engines. A number of advantages explain their popularity:

1. they can be strictly band-limited,

2. they allow the signal power to be placed exactly at the desired frequencies,

3. they are periodic signals,

4. good signal to noise ratio can be achieved by tuning the phases so as to minimise the crest factor of the signal (ratio of peak to rms values).

Provided the multisine is periodic and the data are collected for an integer number of periods, finite time effects can be neglected and application of the Fourier transform to equation (6) leaves the following relations in the frequency domain:

$$
Y\left(\omega_{k}\right)=G_{u}\left(j \omega_{k}, \theta\right) U\left(\omega_{k}\right)+E\left(\omega_{k}\right) \quad k=1,2, \ldots, n_{\omega_{k}}
$$

where the Fourier transform of $e(t)$ is a complex normal distribution $E(\omega) \in \mathcal{N}(0, \Lambda)$, see the appendix for definition and properties. Both the real and imaginary parts are independent and 
jointly normally distributed with zero mean and covariance matrix $\Lambda / 2$.

As a consequence, the Fourier transform of the measurements is distributed according to:

$$
Y\left(\omega_{k}\right) \in \mathcal{N}\left(G_{u}\left(j \omega_{k}, \theta\right) U_{k}\left(\omega_{k}\right), \Lambda\right)
$$

Following a maximum likelihood approach, the estimated health parameters are the solution of the following optimisation problem:

$$
\hat{\theta}=\arg \min _{\theta}\{V(\theta)\}
$$

where the objective function $V(\theta)$ is given by (see [17] for the complete derivation):

$$
V(\theta)=\sum_{k=0}^{n_{\omega}}\left\|Y\left(\omega_{k}\right)-G_{u}\left(i \omega_{k}, \theta\right) U\left(\omega_{k}\right)\right\|^{2}
$$

It is important to note that the sum obviously extends over all the frequencies contained in the multisine input, but integrates also the static component of the total signal which is the only piece of information processed in steady-state methods.

Figure 1 illustrates this issue for a sample single sine wave added to a constant fuel flow. The degradation affecting the engine modifies both static and dynamic characteristics of the output signal: the average level on one hand and the amplitude and phase on the other hand. The frequency is not affected due to the linearity assumption.

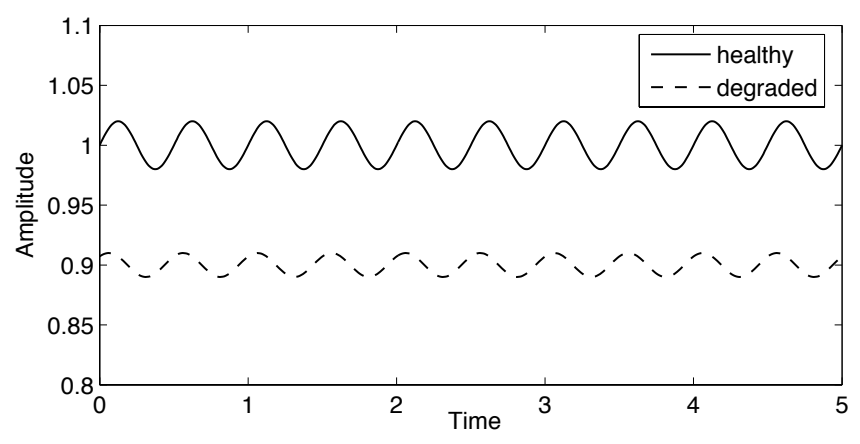

Figure 1. CONTRIBUTION OF THE STATIC AND DYNAMIC PARTS OF THE MEASUREMENT SIGNAL

\section{Assessing the quality of the estimator}

A traditional way of assessing the performance of an estimator is to compute its Cramer-Rao lower bound [21]. Briefly explained, the Cramer-Rao bound introduces a lower limit on the variance of an unbiased estimator. As a reminder, an estimator is unbiased, i.e. $\mathbf{E}(\hat{\boldsymbol{\theta}})=\theta_{\star}$ if the system is of full order. This condition is fulfilled if its Fisher information matrix (FIM, see below) is of full rank.

The Cramer-Rao inequality means that the uncertainty in the health parameters cannot be decreased to an arbitrary level. Mathematically, it writes down:

$$
P \geq M^{-1}
$$

where $P$ is the covariance matrix of the estimates:

$$
P \stackrel{\text { def }}{=} \mathbf{E}\left(\left(\hat{\theta}-\theta_{\star}\right)\left(\hat{\theta}-\theta_{\star}\right)^{T}\right)
$$

$\theta_{\star}$ being the actual value of the health parameters, and $M$ is the FIM defined as:

$$
M \stackrel{\text { def }}{=} \mathbf{E}\left[\sum_{k=0}^{n_{\omega}}\left(\frac{\partial}{\partial \theta} \log p\left(Y\left(\omega_{k}\right), \theta\right)\right)^{*}\left(\frac{\partial}{\partial \theta} \log p\left(Y\left(\omega_{k}\right), \theta\right)\right)\right]
$$

where $p\left(Y\left(\omega_{k}\right), \theta\right)$, the joint probability distribution of the measurements and the parameters, is modelled as a Gaussian law according to (9). The FIM is a square, symmetric and positive definite matrix, the size of which is equal to the number of parameters $n_{\theta}$. It quantifies the amount of information that the observations carry about the unknown parameters.

Introducing the $\left(n_{y} \times n_{\theta}\right)$ matrix $\mathbf{H}\left(\omega_{k}\right)$ as:

$$
\mathbf{H}\left(\omega_{k}\right)=\left[\Psi_{1}\left(j \omega_{k}\right) \Psi_{2}\left(j \omega_{k}\right) \ldots \Psi_{i}\left(j \omega_{k}\right) \ldots \Psi_{n_{\theta}}\left(j \omega_{k}\right)\right]
$$

where $\Psi_{i}\left(j \omega_{k}\right)$ is a notation for:

$$
\Psi_{i}\left(j \omega_{k}\right)=\frac{\partial}{\partial \theta_{i}} G_{u}\left(j \omega_{k}, \theta\right) U_{k}\left(\omega_{k}\right)
$$

and the sensitivity of the model to parameter $i$ being given by:

$$
\begin{aligned}
\frac{\partial}{\partial \theta_{i}} G_{u}\left(j \omega_{k}, \theta\right) & =C_{i}\left(j \omega_{k} I_{n_{x}}-A\right)^{-1} B \\
& -C\left(j \omega_{k} I_{n_{x}}-A\right)^{-1} A_{i}\left(j \omega_{k} I_{n_{x}}-A\right)^{-1} B \\
& +C\left(j \omega_{k} I_{n_{x}}-A\right)^{-1} B_{i} \\
& +D_{i}
\end{aligned}
$$


the FIM becomes:

$$
M=\sum_{k=0}^{n_{\omega}}\left[\mathbf{H}^{*}\left(\omega_{k}\right) \mathbf{H}\left(\omega_{k}\right)\right]
$$

Around a given operating point, the FIM depends solely on the features of the input signal $u(t)$, which are the vector of frequencies $\omega_{k}$ and the associated amplitudes $\alpha_{k}, k=1, \ldots, n_{\omega}$. For the purpose of assessing the improvement in estimation quality brought by a transient signal, it is convenient to summarise the FIM to a scalar measure. It is a standard way to proceed in design of experiments, e.g. [22], where the following three metrics are commonly used:

- the determinant of the FIM, equal to the product of the singular values. It is inversely proportional to the volume of the confidence ellipsoid of the estimates,

- the trace of the inverse of the FIM, equal to the sum of the singular values. It is a measure of the average variance of the estimates,

- the smallest singular value of the FIM is intimately related to the variance of the least-well estimated parameter direction.

\section{APPLICATION OF THE METHOD \\ Engine Layout}

A large bypass ratio mixed-flow turbofan is selected to illustrate the theoretical developments. The engine performance model has been developed in the frame of the OBIDICOTE ${ }^{1}$ project and is detailed in [23].

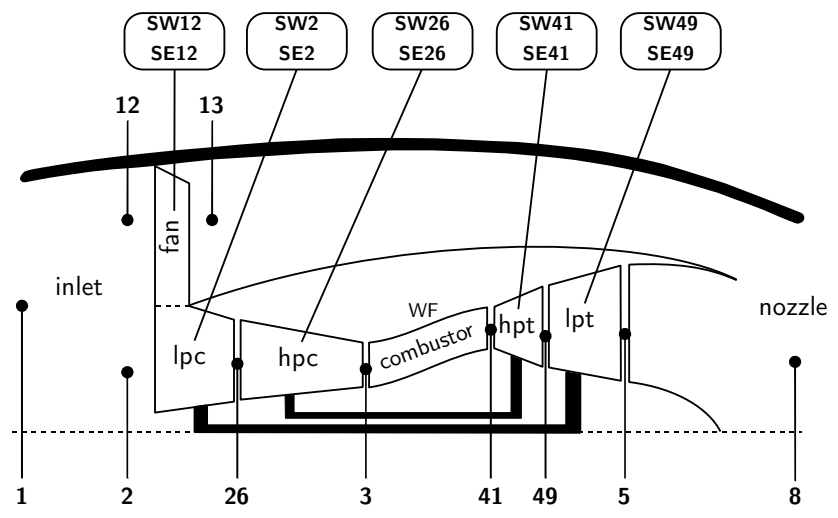

Figure 2. TURBOFAN LAYOUT WITH STATION NUMBERING AND HEALTH PARAMETERS LOCATION

\footnotetext{
${ }^{1}$ A Brite/Euram project for On-Board Identification, Diagnosis and Control of Turbofan Engine
}

A schematic of the engine is sketched in figure 2 where the location of the ten health parameters and the station numbering are also indicated. The engine is open-loop controlled by setting directly the value of the fuel flow $W_{F}$.

A dynamic model is available in the state-space form specified by equations (1). It is able to simulate the shaft dynamics as well as the heat transfers between the working fluid and the components of the gas generator (HPC, combustor, HPT). The present study considers small input signals so that the dynamics of the linearised model is restricted to the mechanical inertia of the spools. The associated state variables are hence the rotational speeds of the low- and high-pressure shafts.

The sensor suite selected to monitor the engine condition is representative of the instrumentation available on-board contemporary turbofan engines and is detailed in table 1 where the nominal accuracy of each sensor is also reported.

Table 1. GAS-PATH INSTRUMENTATION (uncertainty is three times the standard deviation)

\begin{tabular}{lll} 
Label & Description & Uncertainty \\
\hline$P 13$ & fan outlet total pressure & $\pm 100 \mathrm{~Pa}$ \\
$T 26$ & LPC outlet total temperature & $\pm 2 \mathrm{~K}$ \\
$P 3$ & HPC outlet total pressure & $\pm 5000 \mathrm{~Pa}$ \\
$T 3$ & HPC outlet total temperature & $\pm 2 \mathrm{~K}$ \\
$N_{l p}$ & low pressure spool speed & $\pm 6 \mathrm{rpm}$ \\
$N_{h p}$ & high pressure spool speed & $\pm 12 \mathrm{rpm}$ \\
$T 49$ & LPT inlet total temperature & $\pm 2 \mathrm{~K}$ \\
\hline
\end{tabular}

The operating conditions are sea-level static, standard day (ISA-SLS), and the steady-state fuel flow $u_{0}$ corresponds to maximum continuous regime. The baseline values of the health parameters $\theta_{0}$ are equal to one.

\section{Set-up of the parametric study}

It has been previously mentioned that the FIM depends on the characteristics of the input signal. This calls for a parametric study of these features on one of the scalar metrics derived from the FIM. For sake of simplicity, the input signal is limited to a steady-state fuel flow perturbed by a sine wave:

$$
u(t)=u_{0} \cdot\left(1+\alpha_{1} \cos \left(\omega_{1} t\right)\right)
$$

so that the input signal is characterised by two parameters: the relative amplitude $\alpha_{1}$ and the frequency $\omega_{1}$ of the sine wave. 
The experience of Evans et al. in system identification of jet engines, see [20], serves as a guideline to define sound intervals for both parameters. As far as the frequency $\omega_{1}$ is concerned, it is reported that the typical bandwidth for a linear frequency response function of a jet engine is 0 to $1 \mathrm{~Hz}$. The range selected here extends from $10^{-3}$ (almost static) to $10 \mathrm{~Hz}$. This choice is motivated by the fact that gas turbines usually have well damped dynamics. The sampling frequency of the engine control system also sets an upper bound on the frequency interval and rarely exceeds $50 \mathrm{~Hz}$.

Two conflicting requirements govern the choice of the amplitude $\alpha_{1}$. On one hand, the higher the amplitude of the input signal, the better the signal to noise ratio. On the other hand, the gas turbine has a non-linear behaviour and the amplitude should therefore be limited to ensure the validity of the linearised model. In [20], it is stated that a $10 \%$ amplitude of the fuel flow is a good trade-off between both objectives. Therefore, the interval considered here ranges between $0.01 \%$ (tiny amplitude) and $10 \%$.

\section{Validity of the linearised engine model}

Before performing the parametric study, it is safe to check the validity of the linearised engine model over the range of possible input signals used in this study with respect to the two assumptions made earlier.

The first assumption is that the behaviour of the engine can be approximated as linear with a reasonable accuracy. A fuel flow wave of type (19) was applied to both models (linear and non-linear). The frequency of the wave was set at $5 \mathrm{~Hz}$ and the amplitude was varied along the interval defined above. Figure 3 shows the root mean square error for each measurement, computed over 5 periods of the input signal and normalised with respect to the standard deviation of the sensor. As expected, the discrepancy between both models grows as the amplitude $\alpha_{1}$ of the wave increases. However, for the largest amplitude considered here the error due to linearisation lies between $0.01 \%$ and $0.1 \%$, which is still fairly acceptable.

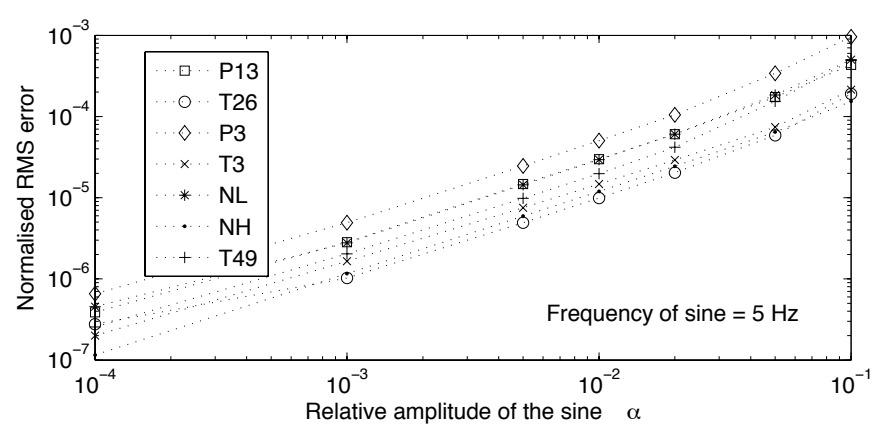

Figure 3. EFFECT OF THE NON-LINEARITY IN THE ENGINE MODEL
The second assumption is that the heat transfers play no significant role on the engine response. This time, a fuel flow wave with a frequency of $5 \mathrm{~Hz}$ and an amplitude of $1 \%$ was applied to the full non-linear model that includes shaft dynamics and heat transfers and to the linearised model based on spool dynamics only. Figure 4 shows the root mean square error for each measurement, defined as in figure 3 . It can be seen that the magnitude of the discrepancy is on the order of $0.02 \%$ for all sensors but $T 49$, which is again acceptable. The maximum error occurs on $T 49$ and could be explained by the cumulative effect on the errors caused by neglecting the heat soakage in the components of the gas generator.

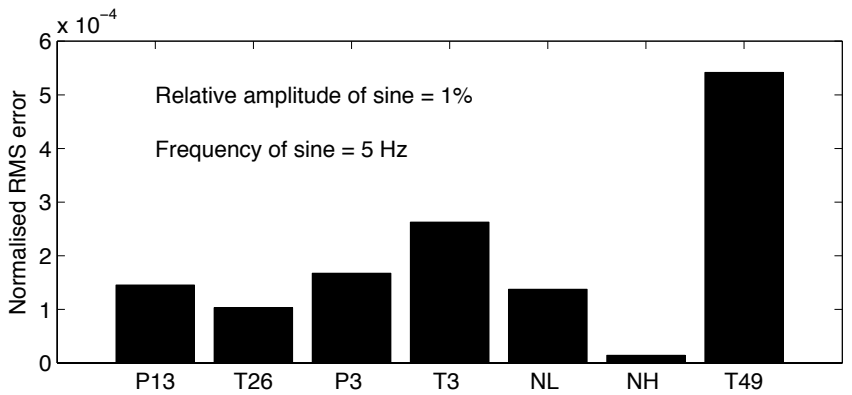

Figure 4. EFFECT OF THE HEAT TRANSFERS IN THE ENGINE MODEL

\section{Results of the parametric study}

From the analysis of figures 3 and 4, it can be concluded that the linearised model represents the behaviour of the "real" engine with a sufficient accuracy. The parametric study can then be conducted. It consists in computing the FIM over a grid of $\left(\omega_{1}, \alpha_{1}\right)$ couples. As the input signal is composed of a static part and a single sine, the computation of the FIM with equation (18) implies a sum of two terms. The frequency and relative amplitude of each term are recalled in table 2 . Note that even if each term in the sum is rank deficient, the FIM may be of full rank, similarly to what happens in multi-point steady-state analysis, see [6].

Table 2. COMPONENTS OF THE FISHER INFORMATION MATRIX

\begin{tabular}{ccc}
$\mathrm{k}$ & $\begin{array}{c}\text { Frequency } \\
\omega_{k}\end{array}$ & $\begin{array}{c}\text { Relative amplitude } \\
U\left(\omega_{k}\right) / u_{0}\end{array}$ \\
\hline 0 & 0 & 1 \\
1 & $\omega_{1}$ & $\alpha_{1}$ \\
\hline
\end{tabular}

Figures 5 and 6 show respectively the evolution of the rank and of the condition number of the FIM over the definition space of the input signal. Basically, the condition number gives the 
number of health parameters (or linear combinations thereof) that can be estimated, on average, without any bias. For very small amplitudes and/or low frequencies, the rank is equal to 7, the number of measurements. The same result is obtained for a single-point, steady-state method. As soon as the amplitude and frequency increase, so does the rank to reach a value of 10 , the number of health parameters. At the same time, the condition number of the FIM becomes finite. These figures are a first major indication of the improvement achieved by a transient analysis, in this case with a periodic input signal, versus a steady-state, single-point one.

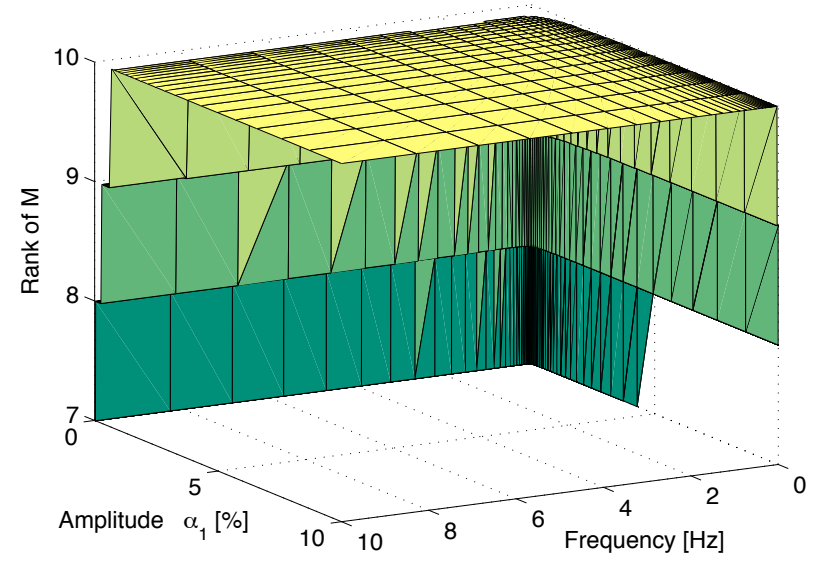

Figure 5. RANK OF THE FIM VERSUS FREQUENCY AND AMPLITUDE OF THE INPUT SIGNAL

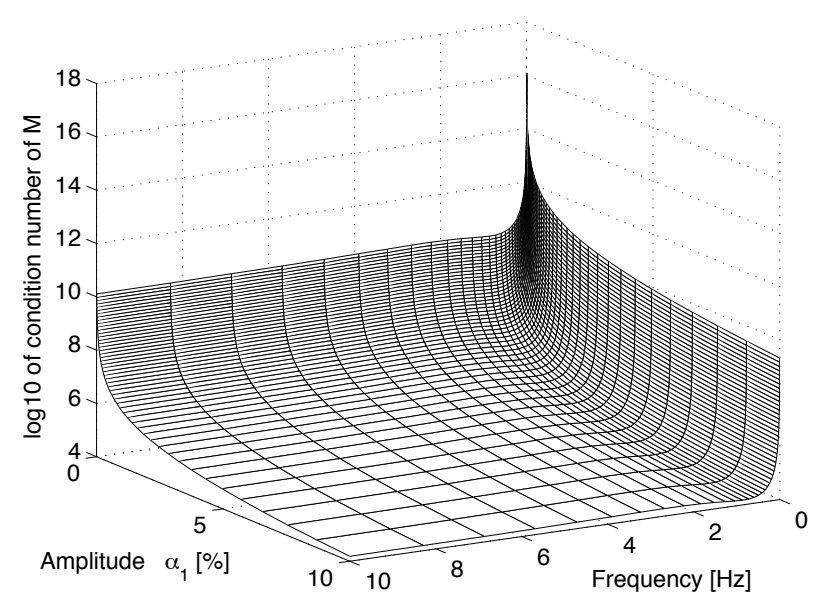

Figure 6. CONDITION NUMBER OF THE FIM VERSUS FREQUENCY AND AMPLITUDE OF THE INPUT SIGNAL

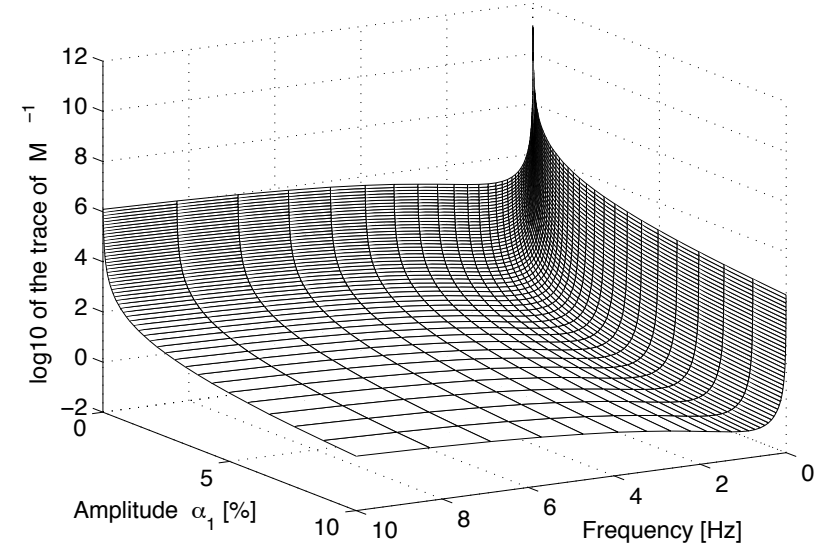

Figure 7. TRACE OF THE INVERSE OF THE FIM VERSUS FREQUENCY AND AMPLITUDE OF THE INPUT SIGNAL

The trace of the inverse of the FIM is selected as an appropriate metric to assess quantitatively the improvement. Remember that the trace is an image of the average variance of the estimates. Figure 7 shows the logarithm of the trace over the $\left(\omega_{1}, \alpha_{1}\right)$ grid. It can be seen that in the vicinity of the $(0,0)$ corner, which represents the classical, pure steady-state case, the value of the trace tends to infinity, which means that the uncertainty in some of the health parameters is very large. This is caused by the underdetermined nature of the estimation problem in that case. At very low frequencies and amplitudes, the magnitude of the trace remains pretty large, which is in accordance with the fact that the FIM is not of full rank for these features of the input signal. A large drop in the trace is witnessed as soon as the rank of the FIM is equal to 10. At a given frequency, it is easily seen that the metric decreases in a monotonic way as the amplitude of the wave increases. This behaviour results from a continuous improvement in the signal to noise ratio.

It is interesting to note the large similarity between figures 6 and 7 which lead to a conclusion previously stated: a transient signal contains more information about the health condition of the engine than a steady-state one.

The evolution of the metric with the frequency at a fixed amplitude looks somewhat more complex. To have a better view of the situation, figure 8 depicts the evolution of the figure of merit with respect to the frequency of the sine for 3 particular amplitudes of $1 \%$ (plain line), 5\% (dashed line) and 10\% (dashdotted line). The behaviour is essentially the same for all three amplitudes.

First, a sharp decrease is noticed as the frequency increases. The trace is reduced by almost two orders of magnitude per decade between $10^{-3}$ and $10^{-1} \mathrm{~Hz}$. As the frequency increases between 0.1 and $1 \mathrm{~Hz}$, the slope decreases and the trace presents a subtle minimum at a frequency of $0.869 \mathrm{~Hz}$, materialised by 
the dotted line in figure 8. Note that the frequency at which the minimum occurs is the same for the three amplitudes. This hints at the fact that the "optimal" frequency is conditioned by the system's properties. Past the minimum, the trace increases slightly, then stabilises in the last portion of the frequency range under investigation. The difference in information content, in terms of the cost function, just above the optimal frequency is very small when a single frequency is used. The amplitude of the signal, as noticed previously, has a far larger influence: about two orders of magnitudes can be gained by switching from a one-percent to a ten-percent amplitude.

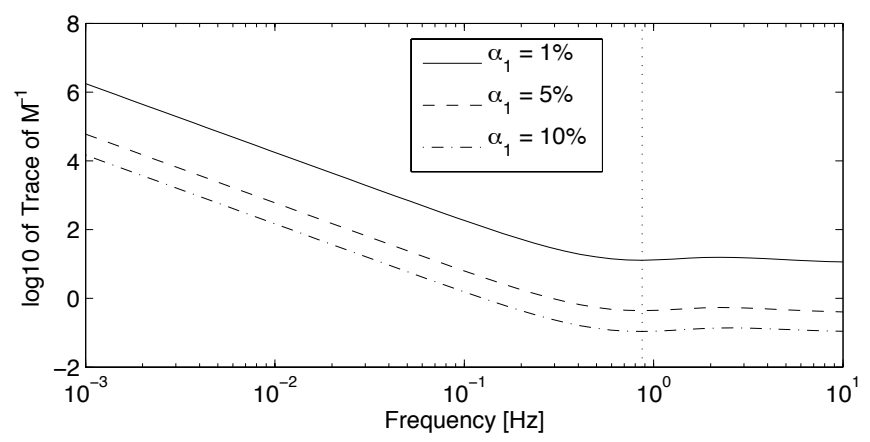

Figure 8. TRACE OF THE INVERSE OF THE FISHER INFORMATION MATRIX VERSUS THE FREQUENCY AT PARTICULAR AMPLITUDES - This graph is extracted from figure 7 , the frequency axis is here on a logarithmic scale

Figure 9 depicts the gain of the transfer function of each sensor versus frequency. It gives the ratio between the amplitude of the output signal and the input signal at a particular frequency. The spool speeds have the typical behaviour of a first order system, the magnitude decreases continuously above the cutoff frequency. For the pressure and temperature measurements, a plateau is observed at high frequencies. This is due to a truncated modelling that does not take into account the gas dynamics nor the sensor dynamics. The bandwidth of the temperature sensors is typically in the range of the Hertz while the bandwidth of the pressure sensors is somewhat larger, albeit depends largely on the installation.

The two poles of the system are located respectively at 0.479 $\mathrm{Hz}$ and $0.713 \mathrm{~Hz}$. In the case of a single sine wave, the optimal frequency of the input signal, materialised by the plain line, is located at $0.869 \mathrm{~Hz}$ and is quite close to the fastest pole.

Figure 10 shows the singular value of the system versus frequency. Loosely speaking, it represent the "overall gain" from the fuel flow to the combined outputs at each frequency. It can be seen that the singular value decreases only moderately as the frequency increases. It is due to the high frequency gains of $T 3$, $P 3$ and $T 49$ (see figure 9). It means that the loss in signal to noise

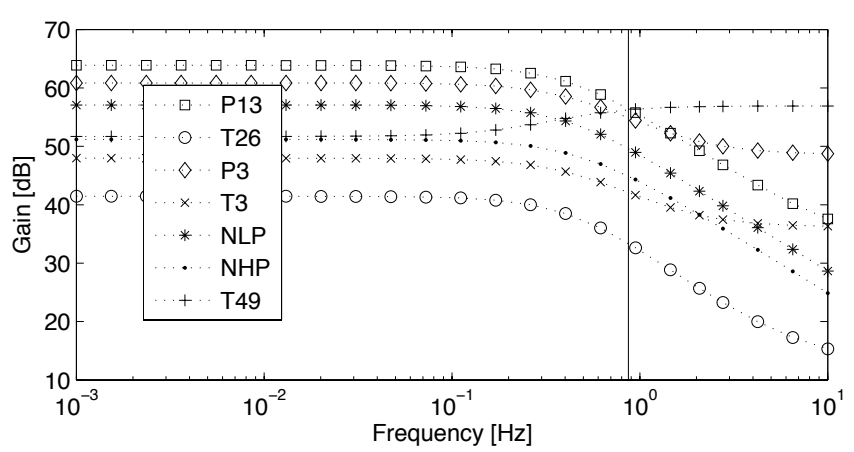

Figure 9. GAIN OF THE TRANSFER FUNCTION BETWEEN FUEL FLOW AND EACH SENSOR VERSUS FREQUENCY - the dotted line represents the optimal frequency

ratio is limited for high frequency signals. The singular value plot provides thereby an explanation to the modest increase in the trace of the inverse of the FIM past the optimal frequency in figure 8 . It is anticipated tha the introduction of sensor dynamics would deteriorate the singular value at high frequency and would translate into a more distinct minimum in figure 8 .

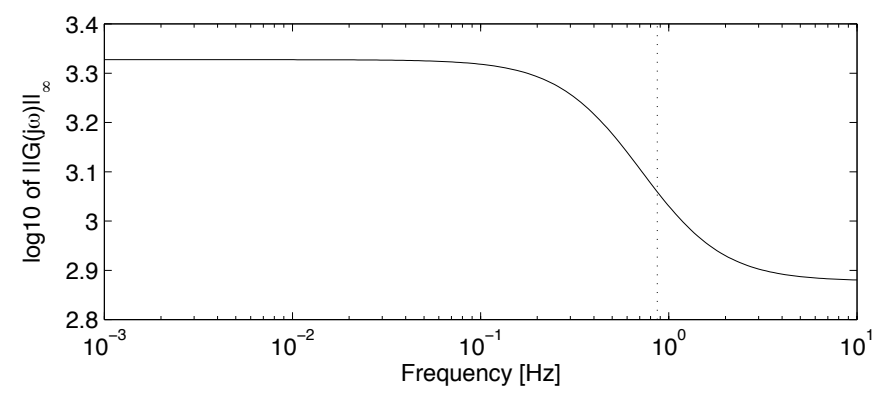

Figure 10. SINGULAR VALUE OF THE SYSTEM VERSUS FREQUENCY- the dotted line represents the optimal frequency

\section{DISCUSSION}

The results of the parametric study have underlined the improvement in the quality of the estimates that can be expected from the processing of transient signals. To complete the analysis of the results, additional questions that call for complementary exploration of the present topic are discussed below.

The first point concerns the practical usefulness of an estimation tool processing the transient signal used in this study. A possible application is the assessment of the baseline performance of each new engine coming out of the production line during the acceptance testing. With a frequency of roughly $1 \mathrm{~Hz}$, several periods of the sine wave can be recorded in a relatively short time (a few dozens of seconds) and averaged to filter out the measurement noise. Such a practice has already been fol- 
lowed by Evans et al. [20] in their experiments on identification of dynamic models of a jet engine and could well be transferred to the present application.

The technique could also be applied to in-service engines. The data could be recorded after each landing for instance. However, it would be desirable to run the engine at a lower power setting for several reasons (e.g. environmental, economics, safety). The influence of the mean power setting on the estimator quality should be studied as it directly affects the signal to noise ratio. As the deterioration evolves with the engine's cycles, the linear engine model should also be updated regularly around the most recent estimated health condition to guarantee its validity.

The influence of the non-linearity and of the neglected heat soakage dynamics has been shown negligible on the model response for the class of input signals considered in the study. The engine was modelled as an open-loop system, and controlled by imposing the perturbed fuel flow directly. In practice however, the engine needs to work within operating constraints and a closed-loop control system is employed. The engine is piloted through a thrust setting parameter (e.g. fan speed or engine pressure ratio). In closed-loop control, the measurement noise is correlated with the input signal (the fuel flow) and the dynamic model should be adapted with a relevant noise model. Reference [24] addresses these specific issues of closed-loop identification.

The present analysis was focused on the case of an input signal consisting of a constant fuel flow perturbed by a single sine wave, but can readily cover a broader spectrum provided the linearity assumption is not violated. Other small transient signals could be analysed by first decomposing them in a series of sines and then performing the present analysis. However, the passage from time to frequency domain with the Fourier transform, which can be regarded as the core of the proposed analysis, is exact only for strictly periodic signals. Otherwise, finite time effects have to be accounted for, see [17].

The extension to large transient trajectory, for instance a snap acceleration from idle to take-off regime, looks as a challenge so far. The non-linearity of the engine with the power setting has to be taken into account. Linear-parameter varying models [25] might offer an appropriate framework.

\section{CONCLUSION}

The present contribution develops a methodology to evaluate the benefit of using transient data for engine health monitoring. A commercial turbofan serves as an example application. The study focuses on a particular class of input signals consisting of a constant fuel flow perturbed by a sine wave for which the assumption of a linear behaviour of the gas turbine is reasonable. A formulation in the frequency domain is adopted, which is shown to be a convenient framework to efficiently summarise the informational content of the transient estimation problem.
The quality of the estimates is assessed by means of two scalar metrics derived from the Fisher information matrix: its rank and its trace. A parametric study applied to a constant fuel flow perturbed with a single sine wave has shown the existence of an optimal frequency that minimises the trace of the Fisher information matrix. This frequency is close to those associated to the spool dynamics. The amplitude of the sine wave should be as large as possible to enhance the signal to noise ratio while ensuring the validity of the linear model approximation.

\section{REFERENCES}

[1] A. J. Volponi. Foundation of gas path analysis (part i and ii). In von Karman Institute Lecture Series, number 01 in Gas Turbine Condition Monitoring and Fault Diagnosis, Brussels, Belgium, 2003.

[2] L. A. Urban. Gas path analysis applied to engine condition monitoring. In 8th Joint Propulsion Specialist Conference, number AIAA paper 72-1082, 1972.

[3] D. L. Doel. An assessment of weighted-least-squares-based gas path analysis. ASME J. of Eng. for Gas Turbines and Power, 116:336-373, 1994.

[4] P. Dewallef, K. Mathioudakis, and O. Léonard. On-line aircraft engine diagnostic using a soft-constrained kalman filter. In ASME Turbo Expo, number GT2004-53539, Vienna, Austria, 2004.

[5] A. Gulati, D.Taylor, and R.Singh. Multiple operating point analysis using genetic algorithm optimisation for gas turbine diagnostics. In 15th International Symposium on Air Breathing Engines, number 1139, Bangalore, India, 2001.

[6] T. Grönstedt. Identifiability in multi-point gas turbine parameter estimation problems. In ASME Turbo Expo, number GT2002-30020 in Controls, Diagnostics and instrumentation, Amsterdam, Netherlands, 2002.

[7] G. Merrington. Fault diagnosis in gas turbine engines from transient data. ASME J. of Eng. for Gas Turbines and Power, 111:237-243, 1989.

[8] R.W. Cue and D.E. Muir. Engine performance monitoring and troubleshooting techniques for the cf-18 aircraft. ASME J. of Eng. for Gas Turbines and Power, 113(1):11-19, 1991.

[9] R.H. Luppold, G.W. Gallops, L.J. Kerr, and J.R. Roman. Estimating in-flight engine performance variations using Kalman filter concepts. In 25th AIAA/ASME/SAE/ASEE Joint Propulsion Conference, number AIAA-89-2584, Monterey CA, USA, 1989.

[10] T. Grönstedt. Least squares based transient nonlinear gas path analysis. In ASME Turbo Expo, number GT200568717, Reno-Tahoe NV, USA, 2005.

[11] Ph. Kamboukos and K. Mathioudakis. Turbofan engine health assessment by combining steady and transient state aerothermal data. In 7th European Conference on Turbomachinery, number 137, Athens, Greece, 2007. 
[12] S. Ogaji, Y.G. Li, S. Sampath, and R. Singh. Gas path fault diagnosis of a turbofan engine from transient data using artificial neural networks. In ASME Turbo Expo, number GT2003-38423, Atlanta GA, USA, 2003.

[13] S. Sampath, Y.G. Li, S.O.T Ogaji, and R. Singh. Fault diagnosis of a two spool turbo-fan engine using transient data: A genetic algorithm approach. In ASME Turbo Expo, number GT2003-38300, Atlanta GA, USA, 2003.

[14] D. Simon and D. L. Simon. Aircraft turbofan engine health estimation using constrained Kalman filtering. In ASME Turbo Expo, number GT2003-38584, Atlanta GA, USA, 2003.

[15] S. Borguet, P. Dewallef, and O. Léonard. On-line transient engine diagnostics in a Kalman filtering framework. In ASME Turbo Expo, number GT2005-68013, Reno-Tahoe NV, USA, 2005.

[16] N. Sugiyama. Derivation of system matrices from nonlinear dynamic simulation of jet engines. AIAA J. of Guidance, Control and Dynamics, 17(6):1320-1326, 1994.

[17] Lennart Ljung. System Identification, Theory for the User (2nd edition). Prentice Hall Information and System Sciences Series, 1999.

[18] M. Henriksson, S. Borguet, O. Léonard, and T. Grönstedt. On inverse problems in turbine engine parameter estimation. In ASME Turbo Expo, number GT2007-27756, Montréal QC, Canada, 2007.

[19] C. Evans, A. Borrell, and D. Rees. Testing and modeling gas turbines using multisine signals and frequency-domain techniques. ASME J. of Eng. for Gas Turbines and Power, 121:451-457, 1999.

[20] C. Evans, P.J. Fleming, D.C. Hill, J.P. Norton, I. Pratt, D. Rees, and K. Rodriguez-Vazquez. Application of system identification techniques to aircraft gas turbine engines. Control Engineering Practice, 9:135-148, 2001.

[21] C. R. Rao. Linear Statistical Inference and Its Applications. John Wiley \& Sons, New-York, 1973.

[22] C.R. Rojas, J.S. Welsh, G.C. Goodwin, and A. Feuer. Robust optimal experiment design for system identification. Automatica, 43(6):993-1008, 2007.

[23] A. Stamatis, K. Mathioudakis, J. Ruiz, and B. Curnock. Real-time engine model implementation for adaptive control and performance monitoring of large civil turbofans. In ASME Turbo Expo, number 2001-GT-0362, New-Orleans LA, USA, 2001.

[24] U. Forssell and L. Ljung. Closed-loop identification revisited. Automatica, 35(7):1215-1241, 1999.

[25] L. Reberga, D. Henrion, J. Bernussou, and F. Vary. LPV modelling of a turbofan engine. In 16th IFAC world congress on automatic control, volume 16-1, Prague, Czech Republic, 2005.

\section{APPENDIX: The complex Normal distribution}

Let $a$ be a random vector whose elements may take complex values. Its mean and covariance matrix are defined as

$$
\begin{gathered}
m \stackrel{\text { def }}{=} \mathbf{E}(a) \\
\operatorname{Cov}(a) \stackrel{\text { def }}{=} \mathbf{E}\left[(a-m)(a-m)^{*}\right]
\end{gathered}
$$

In this case, the notation $a \in \mathcal{N}(m, C)$ will mean that

1. the real and imaginary part of $a$ are jointly normal,

2. $\mathbf{E}(a)=m$, a complex number

3. $\operatorname{Cov}(a)=C$,

4. the real $\mathfrak{R}(a)$ and imaginary $\mathfrak{I}(a)$ parts are independent,

5. $\operatorname{Cov}(\Re(a))=\operatorname{Cov}(\mathfrak{I}(a))=1 / 2 C$ 